\title{
Relations entre le flux salivaire et le taux élevé des Streptocoques mutans et des Lactobacilles salivaires chez l'enfant diabétique de type 1
}

\author{
Manuel Messa Savi de Tove*, Ramata Bakayoko-Ly, Arthur Koffi N'Guessan \\ Département d'0donto-stomatologie pédiatrique, UFR d'Odonto-stomatologie, Abidjan, Côte d'Ivoire
}

(Reçu le 23 juillet 2012, accepté le 30 juillet 2012)

Mots clés : diabète de type 1 / flux salivaire / Streptocoques mutans salivaires / Lactobacilles salivaires / enfants

Key words: type 1 diabetes mellitus / salivary flow / Streptococcus mutans salivary / Lactobacilli salivary / children

\begin{abstract}
Résumé - Ce travail avait pour but d'examiner les interrelations entre le faible débit salivaire et le nombre élevé des colonies de Streptocoques mutans et de Lactobacilles salivaires chez des enfants ayant un diabète sucré de type 1. L'enquête a été effectuée sur 158 enfants âgés de 5 à 12 ans (128 enfants diabétiques et 30 enfants témoins non diabétiques ne souffrant d'aucune maladie systémique); ils ont été sélectionnés dans une clinique pédiatrique et le centre hospitalier universitaire de Lomé (Togo), spécialisés dans le traitement des enfants diabétiques. Il s'agit d'une étude transversale comparative. Chaque groupe d'enfants a été divisé en deux tranches d'âge : de 5-8 ans et de 9-12 ans. Le débit salivaire a été évalué chez tous les patients, chaque enfant a subi un test salivaire à l'aide du « CTR bactéria de Vivadent » afin de déterminer les colonies salivaires de Streptocoques mutans et de Lactobacilles. Les 128 enfants diabétiques de type I présentent une hyposialie avec un débit salivaire de $0,59 \mathrm{ml} \mathrm{min}^{-1}$ (la valeur normale est de $\left.0,70 \mathrm{ml}_{\text {. }} \mathrm{min}^{-1}\right)$. La salive de ces enfants renferme une quantité importante de colonies de Streptocoques mutans et de Lactobacilles. La formation de plaque dentaire bactérienne est à l'origine de la carie et de l'inflammation des tissus parodontaux chez l'enfant diabétique de type 1 . Des conseils de prophylaxie individuelle doivent être prodigués par le médecin dentiste pédiatrique.
\end{abstract}

\begin{abstract}
Relations between the salivary flow rate and the important of Streptococcus mutans and Lactobacilli salivary, among the diabetic child of type 1 . The aim of this study was to examine the interrelationships between the low salivary flow and the high number of salivary colonies of Streptococcus mutans and of Lactobacilli of children with diabetes mellitus type 1 . The survey was conducted on 158 children between the ages of 5 to 12 years (128 children with diabetes and 30 non-diabetic children witnesses not suffering from any systemic disease); they have been selected in a pediatric clinic and the university hospital center of Lome (Togo), specialized in the treatment of children with diabetes. It is a cross-sectional comparative study. Each group of children has been divided into two age groups of 5-8 years and 9-12 years. The salivary flow was assessed on the 158 patients; each child has suffered a salivary test with the help of the "CTR bacteria of Vivadent" in order to determine the settlements of salivary mutans Streptococci and Lactobacilli. The 128 children with diabetes type 1 present a xerostomy with a salivary flow of $0.59 \mathrm{ml} . \mathrm{min}^{-1}$ (the normal value is $0.70 \mathrm{ml} \cdot \mathrm{min}^{-1}$ ). The saliva of these children contains a significant quantity of colonies of Streptococcus mutans and Lactobacilli. The formation of bacterial plaque is at the origin of the caries and inflammation of the periodontal tissues, among the diabetic child of type I. Prophylactic advice of individual must be provided by pediatrician dentist.
\end{abstract}

*Correspondance : savi_manuel2006@yahoo.fr 
Le diabète insulino-dépendant de type I se définit comme une atteinte progressive des cellules de type $\beta$ du pancréas. Chez l'enfant, c'est la diminution, voire la disparition de la sécrétion d'insuline qui est à l'origine du diabète [1]. Le patient diabétique présente une diminution du flux salivaire par rapport au sujet en bonne santé, ce qui favorise l'accumulation de la plaque dentaire, le développement des caries dentaires et des parodontites, tout en favorisant la prolifération de certaines bactéries salivaires, en particulier les Streptocoques mutans et des Lactobacilles $[6,8,11]$.

L'objectif de cette étude était d'apprécier les modifications quantitatives éventuelles des colonies de Streptocoques mutans et de Lactobacilles salivaires par rapport à la diminution du flux salivaire liée au diabète insulino-dépendant de type I chez l'enfant.

\section{Matériel et méthodes}

\section{Matériel}

\section{Population d'étude}

L'échantillon de cette étude était composé de 128 enfants diabétiques, âgés de 5 à 12 ans. Ils ont été sélectionnés dans une clinique pédiatrique et dans le centre hospitalo-universitaire de Lomé (Togo), spécialisés dans la prise en charge d'enfants diabétiques. L'enquête s'est déroulée en août 2008.

\section{Critères d'inclusion et de non inclusion}

On a retenu seulement les enfants diabétiques de type I, âgés de 5 à 12 ans, n'ayant pas encore reçu des soins médicaux. On n'a pas inclus les enfants:

- présentant une pathologie générale autre que le diabète et recevant une thérapie médicamenteuse ;

- âgés de moins de 5 ans ou de plus 12 ans ;

- ayant un diabète traité et surveillé conformément à un protocole médical standard et ayant reçu des soins préventifs.

\section{Matériel d'étude}

L'instrumentation qui a servi à la réalisation de cette étude était composée de plateaux d'examen, d'un matériel d'analyse du débit salivaire qui comportait des tubes gradués de prélèvement de la salive et le «CTR bactéria de Vivadent»
(CTRbacteria Portion Standard Vivadent Ets. FL. 9494, Schaan, Principauté du Liechtenstein) pour la détermination des colonies de Streptocoques mutans et de Lactobacilles salivaires.

\section{Méthodes}

\section{L'échantillonnage}

Un enrôlement aléatoire des enfants témoins sains et non diabétiques a été réalisé dans des centres de santé spécialisés dans le traitement des diabétiques de Lomé (Togo). Les enfants diabétiques étaient recrutés lorsqu'un diabète de type 1 était constaté dans les investigations cliniques. Une autorisation a été obtenue de la part des parents pour la réalisation de ce travail. Il s'agissait d'une enquête épidémiologique, descriptive, transversale intéressant un ensemble de 128 enfants diabétiques et de 30 enfants témoins, non diabétiques. Les paramètres glycémiques et l'hémoglobine glyquée sont contenus dans le dossier médical de chaque enfant.

\section{Analyse salivaire}

La salive au repos des 158 enfants a été recueillie dans un tube de prélèvement stérile de $15 \mathrm{ml}$. Une sialométrie a été réalisée pour montrer les distinctions entre le flux salivaire des enfants diabétiques et des non diabétiques. Le débit salivaire était calculé en $\mathrm{ml}$ par minute $\left(\mathrm{ml} \cdot \mathrm{min}^{-1}\right)$. L'utilisation du CTR bactéria de Vivadent a servi pour évaluer le nombre de Streptocoques mutans et de Lactobacilles dans la salive. C'est un test de diagnostic in vitro. Les deux bandes de gélose bleue et verte sont complètement humectées avec la salive des enfants à l'aide d'une pipette. Après avoir bien refermé le tube, il est conservé dans un incubateur pendant $48 \mathrm{~h}$ à $37^{\circ} \mathrm{C}$. Ensuite la densité des colonies bactériennes a été comparée avec la représentation correspondante de la fiche d'interprétation. Un résultat supérieur à $10^{5}$ bactéries (Streptocoques mutans ou Lactobacilles) par $\mathrm{ml}$ de salive correspond à un risque de carie dentaire élevé ; un résultat inférieur à $10^{5}$ par $\mathrm{ml}$ de salive à un risque de carie faible.

\section{Analyse des données}

La saisie des données et les calculs statistiques ont été effectués sur le logiciel EPI INFO ; le Chi 2 de Pearson a été utilisé. En présence d'une différence significative « $S$ », le degré de signification « $P$ » précise un risque d'erreur $\alpha$ fixé à $5 \%$. 


\section{Résultats}

Tableau I. Répartition des enfants diabétiques et non diabétiques selon l'âge.

Table I. Distribution of children with and without diabetes according to the age.

\begin{tabular}{|l|c|c|c|c|c|c|}
\hline \multirow{2}{*}{ Tranches d'âge } & \multicolumn{2}{|c|}{ Diabétiques } & \multicolumn{2}{c|}{ Non diabétiques } & \multicolumn{2}{c|}{ Total } \\
\cline { 2 - 7 } & $N$ & $\%$ & $N$ & $\%$ & 109 & 69 \\
\hline $5-8$ ans & 84 & 65,6 & 25 & 83,3 & 49 & 31 \\
\hline $9-12$ ans & 44 & 34,4 & 5 & 16,7 & 158 & 100 \\
\hline Total & 128 & 100 & 30 & 100 & 158 \\
\hline
\end{tabular}

Tableau II. Débit salivaire des enfants diabétiques et non diabétiques.

Table II. Salivary flow rate of children with and without diabetes.

\begin{tabular}{|c|c|c|c|}
\hline \multicolumn{2}{|c|}{ Diabétiques } & \multicolumn{2}{|c|}{ Non diabétiques } \\
\hline$N$ & Débit salivaire en $\mathrm{ml} \cdot \mathrm{min}^{-1}$ & $N$ & Débit salivaire en $\mathrm{ml} \cdot \mathrm{min}^{-1}$ \\
\hline 128 & $0,59 \pm 0,25$ & 30 & $0,70 \pm 1,58$ \\
\hline
\end{tabular}

Tableau III. Répartition des enfants diabétiques et non diabétiques en fonction du débit salivaire, puis du nombre des colonies de Streptocoques mutans et de Lactobacilles.

Table III. Distribution of children with and without diabetes in function of the salivary flow and then of the colonies number of Streptococcus mutans and Lactobacilli.

\begin{tabular}{|c|c|c|c|c|c|c|c|c|}
\hline \multicolumn{4}{|c|}{ Diabétiques } & \multicolumn{4}{|c|}{ Non diabétiques } & \multirow{3}{*}{$\mathrm{P}$} \\
\hline \multicolumn{4}{|c|}{ Débit salivaire $=0,59 \mathrm{ml} \cdot \mathrm{min}^{-1}$} & \multicolumn{4}{|c|}{ Débit salivaire $=0,70 \mathrm{ml} \cdot \mathrm{min}^{-1}$} & \\
\hline \multicolumn{2}{|c|}{$5-8$ ans } & \multicolumn{2}{|c|}{$9-12$ ans } & \multicolumn{2}{|c|}{$5-8$ ans } & \multicolumn{2}{|c|}{$9-12$ ans } & \\
\hline \multicolumn{8}{|c|}{ Streptocoques mutans salivaires } & \\
\hline \multicolumn{4}{|c|}{$>10^{5} / \mathrm{ml}$ de salive } & \multicolumn{4}{|c|}{$<10^{5} / \mathrm{ml}$ de salive } & \\
\hline$N$ & $\%$ & $N$ & $\%$ & $N$ & $\%$ & $N$ & $\%$ & \multirow{2}{*}{$\mathrm{S}$} \\
\hline 43 & 51,2 & 35 & 79,5 & 14 & 56 & 5 & 100 & \\
\hline \multicolumn{8}{|c|}{ Lactobacilles salivaires } & \\
\hline \multicolumn{4}{|c|}{$>10^{5} / \mathrm{ml}$ de salive } & \multicolumn{4}{|c|}{$<10^{5} / \mathrm{ml}$ de salive } & \\
\hline$N$ & $\%$ & $N$ & $\%$ & $N$ & $\%$ & $N$ & $\%$ & \multirow{2}{*}{$\mathrm{S}$} \\
\hline 41 & 48,8 & 9 & 20,5 & 11 & 44 & 0 & 0 & \\
\hline
\end{tabular}

\section{Discussion}

Sur 128 enfants diabétiques, $84(65,6 \%)$ enfants sont dans la tranche de 5 à 8 ans (Tab. I); ceci confirme que le diabète insulino-dépendant de type I de l'enfant est une affection précoce et qu'elle apparaît le plus souvent dans cette tranche d'âge [3]. Les enfants diabétiques présentent un débit salivaire moins important $\left(0,59 \mathrm{ml} \mathrm{min}^{-1}\right)$ que les enfants témoins non diabétiques $\left(0,70 \mathrm{ml} \cdot \mathrm{min}^{-1}\right)$ (Tab. II). La diminution de la sécrétion salivaire est l'une des principales conséquences du diabète insulino-dépendant de type I chez l'enfant. Lopez et al. [5] et Wu et al. [12] ont montré que les enfants diabétiques présentent une fréquence carieuse plus importante que les enfants non diabétiques, une augmentation du taux de glucose salivaire, une hyposialie et une diminution du pH salivaire ; Wu et al. [12] ont souligné que la salive constitue un élément important pour le maintien d'une bonne santé bucco-dentaire. 
La carie est une maladie infectieuse dont l'apparition est favorisée par un taux anormalement élevé de certaines bactéries, plus particulièrement les Streptocoques mutans et les lactobacilles qui sont respectivement impliqués dans l'initiation de la lésion carieuse et dans sa progression. La diminution du débit salivaire, une alimentation riche en sucres et bien sûr une mauvaise bucco-hygiène dentaire constituent des facteurs favorisants bien connus.

Les tests bactériologiques pour la quantification des bactéries cariogènes (Streptocoques mutans et Lactobacilles) dans la salive permettent d'évaluer le risque carieux des enfants. La présence de Streptocoques mutans est d'autant plus fréquente que l'âge des sujets augmente (Tab. III). La différence de pourcentages entre les deux tranches d'âge pourrait s'expliquer par:

- les infections dentaires et les gingivites qui tendent à perturber l'hygiène bucco-dentaire ;

- le fait que l'enfant soit quotidiennement en rapport avec des Streptocoques mutans et des Lactobacilles car il consomme des produits fermentés par ces bactéries ;

- l'hérédité qui aurait aussi un rôle significatif dans l'incidence et la progression des caries en influençant la colonisation bactérienne buccale [2] ;

- l'influence des facteurs sociodémographiques et économiques liés à la famille, la fréquentation par l'enfant d'un cabinet dentaire, la fréquence de la consommation d'aliments cariogènes et le niveau d'hygiène bucco-dentaire influencent la composition quantitative de la flore bactérienne buccale [4] ;

- l'environnement social qui conditionne les habitudes alimentaires, peut favoriser la prolifération des Streptocoques mutans et des Lactobacilles salivaires [7].

Comparativement aux enfants non diabétiques du même âge, chez les 128 enfants ayant un diabéte de type I âgés de 5 à 12 ans et présentant une diminution du flux salivaire $\left(0,59 \mathrm{ml} \cdot \mathrm{min}^{-1}\right)$, les colonies de Streptocoques mutans et de Lactobacilles sont de façon significative $\left(P=10^{-3}\right)$ plus souvent supérieures à $10^{5} \mathrm{par} \mathrm{ml}$ de salive (Tab. III). Cette observation a déjà été faite par Twetman et al. [10] et Siudikiene et al. [9]. Pour ces auteurs, les enfants ayant un diabète insulinodépendant de type I ont tendance à présenter une diminution du débit salivaire et leur salive à contenir un nombre élevé de colonies de Streptocoques mutans et de Lactobacilles. Ceci semble constituer un facteur favorisant important pour le développement des caries dentaires et des parodontopathies.

\section{Conclusion}

Chez l'enfant, le diabète de type I provoque des perturbations $\mathrm{du} \mathrm{pH}$ et du pouvoir tampon salivaires. De plus, les phénomènes infectieux inhérents à la maladie et la mauvaise hygiène bucco-dentaire entrainent l'apparition de caries dentaires dues à la plaque bactérienne et à l'inflammation gingivale. S'il est primordial pour le médecin diabétologue d'équilibrer le diabète, ce dernier doit aussi se préoccuper de l'état bucco-dentaire des ces enfants diabétiques. Il devrait systématiquement les confier au chirurgien dentiste afin qu'ils reçoivent des conseils individuels de prophylaxie :

- brossage des dents après chaque repas ;

- utilisation d'un dentifrice adapté à l'atteinte gingivale ;

- réalisation d'une hygiène bucco-dentaire rigoureuse.

Cette prévention permet de limiter le nombre de caries, de ralentir la progression de la maladie parodontale et d'entreprendre rapidement leur traitement.

\section{Conflits d'intérêt : aucun}

\section{Références}

1. Chiu KC, Chu A, Go VL, Saad MF. Hypovitaminosis D is associated with insulin resistance and beta cell dysfunction. Am J Clin Nutr 2004;79:820-5.

2. Corby PM, Lyons-Weiler J, Bretz WA, Hart TC, Aas JA, Bomenna T, Goss J, Corby AL, Junior HM, Weyant RJ, Paster BJ. Microbial risk indicators of early childhood caries. J Clin Microbiol 2005;43:5753-9.

3. Edblad E, Lundin SA, Sjodin B, Aman J. Caries and salivary status in young adults with type 1 diabetes. Swed Dent J 2001;25:53-60.

4. Koko J, Ategbo S, Ateba Ngoa U, Moussavou A. Étude épidémiologique de la carie dentaire en milieu scolaire à Libreville, Gabon. Clin Mother Child Health 2009;6:1065-73.

5. Lopez ME, Colloca ME, Paez RG, Schallmach JN, Koss MA, Chervonagura A. Salivary characteristics of type 1 diabetes in children. Braz Dent J 2003;14:26-31.

6. Moore PA, Guggenheimer J, Etzel KR, Weyant RJ, Orchard T. Type 1 diabetes mellitus, xerostomia, and salivary flow rates. Oral Surg Oral Med Oral Pathol Oral Radiol Endod 2001;92:281-91.

7. Pine C. International perspectives on targeting caries prevention. World congress on preventive dentistry, Beijing, China. 2000;7:47-53.

8. Rai K, Hegde AM, Kamath A, Shetty S. Dental caries and salivary alterations in type I diabetes. J Clin Pediatr Dent 2011;36:181-4.

9. Siudikiene J, Machiulskiene V, Nyvad B, Tenovuo J, Nedzelskiene I. Dental caries and salivary status in children with type 1 diabetes mellitus, related to the metabolic control of the disease. Eur J Oral Sci 2006;114:8-14.

10. Twetman S, Aronsson S, Björkman S. Mutans Streptococci and Lactobacilli in saliva from children with insulin dependent diabetes mellitus. Oral Microbiol Immunol 1989;4:165-8.

11. Twetman S, Johansson I, Birkhed D, Nederfors T. Caries incidence in young type 1 diabetes mellitus patients in relation to metabolic control and caries associated risk factors. Caries Res 2002;36:315.

12. Wu Katie $P$, Jyh-Yuh K, Chia-Ying C, Chia-Ling C, Tsong-Long H, Ming-Yen C. Relationship between unstimulated salivary flow rate and saliva composition of healthy children in Taiwan. Chang Gung Med J 2008;31:281-6. 